\title{
Adaptive optics tracking and pushing system for space debris manoeuvre
}

\section{Marcus Lingham, Doris Grosse, Francis Bennet, Mark Blundell, Amy Chan, et al.}

Marcus Lingham, Doris Grosse, Francis Bennet, Mark Blundell, Amy Chan, Michael Copeland, Celine d'Orgeville, Michael Ellis, Antony Galla, Yue Gao, Luke Gers, John Hart, Ellen Houston, Visa Korkiakoski, lan Price, Emily Rose Rees, Francois Rigaut, Ian Ritchie, Craig Smith, Tony Travouillon, Annino Vaccarella, Yanjie Wang, James Webb, "Adaptive optics tracking and pushing system for space debris manoeuvre

," Proc. SPIE 10703, Adaptive Optics Systems VI, 1070300 (10 July 2018); doi: $10.1117 / 12.2313181$

Event: SPIE Astronomical Telescopes + Instrumentation, 2018, Austin, Texas, United States 


\title{
Adaptive optics tracking and pushing system for space debris manoeuvre
}

\author{
Marcus Lingham ${ }^{\mathrm{a}, \mathrm{b}}$, Doris Grosse ${ }^{\mathrm{a}, \mathrm{b}}$, Francis Bennet ${ }^{\mathrm{a}, \mathrm{b}}$, Mark Blundell ${ }^{\mathrm{b}, \mathrm{c}}$, Amy Chan ${ }^{\mathrm{b}, \mathrm{c}}$, \\ Michael Copeland $^{\mathrm{a}, \mathrm{b}}$, Celine d'Orgevilla ${ }^{\mathrm{a}, \mathrm{b}}$, Michael Ellis ${ }^{\mathrm{a}, \mathrm{b}}$, Antony Galla ${ }^{\mathrm{a}, \mathrm{b}}$, Yue Gao ${ }^{\mathrm{b}, \mathrm{c}}$, Luke \\ Gers $^{\mathrm{a}, \mathrm{b}}$, John Harta ${ }^{\mathrm{a}, \mathrm{b}}$, Ellen Houston ${ }^{\mathrm{a}, \mathrm{b}}$, Visa Korkiakoski ${ }^{\mathrm{a}, \mathrm{b}}$, Ian Price Pra $^{\mathrm{a}, \mathrm{b}}$, Emily Rose Rees ${ }^{\mathrm{a}, \mathrm{b}}$, \\ Francois Rigaut $^{\mathrm{a}, \mathrm{b}}$, Ian Ritchie ${ }^{\mathrm{b}, \mathrm{c}}$, Craig Smith ${ }^{\mathrm{b}, \mathrm{c}}$, Tony Travouillon ${ }^{\mathrm{a}, \mathrm{b}}$, Annino Vaccarella ${ }^{\mathrm{a}, \mathrm{b}}$, \\ Yanjie Wang ${ }^{\mathrm{b}, \mathrm{c}}$, and James Webb ${ }^{\mathrm{b}, \mathrm{c}}$ \\ ${ }^{a}$ Research School of Astronomy and Astrophysics, Australian National University, Canberra, \\ ACT 2611, Australia \\ ${ }^{\mathrm{b}}$ Space Environment Research Centre (SERC Limited), Mount Stromlo Observatory, Weston \\ Creek, ACT 2611, Australia \\ ${ }^{\mathrm{c}}$ EOS Space Systems, Mount Stromlo Observatory, Cotter Road, Weston Creek, ACT 2611, \\ Australia
}

\begin{abstract}
As space debris in lower Earth orbits are accumulating, techniques to lower the risk of space debris collisions must be developed. Within the context of the Space Environment Research Centre (SERC), the Australian National University (ANU) is developing an adaptive optics system for tracking and pushing space debris. The strategy is to pre-condition a laser launched from a $1.8 \mathrm{~m}$ telescope operated by Electro Optics Systems (EOS) on Mount Stromlo, Canberra and direct it at an object to perturb its orbit. Current progress towards implementing this experiment, which will ensure automated operation between the telescope and the adaptive optics system, will be presented.
\end{abstract}

Keywords: Adaptive optics, space situational awareness

\section{INTRODUCTION}

Low Earth orbit (LEO) is an altitude band ranging from approximately 200-2000 $\mathrm{km}$ that is important for the functioning of today's society. Man-made satellites orbiting in this regime serve to assist technologies in fields such as communications and imaging. Recent space activity has seen an increase in the number of satellites occupying this space due to higher demand for their applications. This has entailed a rise in the number of debris objects in LEO which includes material leftover from previous launches, dead satellites, and other mission related objects. As a consequence the probability of a collision event between two objects in LEO has risen.

Considering particles within this regime tend to travel at speeds around $7 \mathrm{~km} / \mathrm{s}$, the case of such a collision can be devastating for the space environment. A collision could result in a cloud of smaller orbiting fragments that were previously constituents of the objects involved in the collision to be released into LEO. A good example of this is the 2009 collision between the Iridium 33 and Cosmos 2251 satellites. $^{1}$ The increased number of objects scales up the likelihood of a further impact which could then lead to a cascading effect first predicted by Kessler. ${ }^{2}$ The circumstance where a high density of fragments renders LEO unusable for satellites is known as the Kessler syndrome.

The main collision avoidance tactic that is currently implemented involves manoeuvring an at-risk satellite to significantly lower the probability of collision with another object. This works predominantly for objects larger than $10 \mathrm{~cm}$ in diameter as they are easier to track. Otherwise satellites can be equipped with Whipple shielding

Further author information: (Send correspondence to Marcus Lingham)

Marcus Lingham: E-mail: marcus.lingham@anu.edu.au, Telephone: +61 261250218

Adaptive Optics Systems VI, edited by Laird M. Close, Laura Schreiber, Dirk Schmidt, Proc. of SPIE Vol. 10703, 1070300 - @ 2018 SPIE CCC code: $0277-786 \mathrm{X} / 18 / \$ 18 \cdot$ doi: $10.1117 / 12.2313181$

Proc. of SPIE Vol. 10703 1070300-1 
in order to reduce the impact from small particles less than $1 \mathrm{~cm}$ in diameter. Therefore a viable strategy for objects ranging 1-10 $\mathrm{cm}$ in diameter would be a necessary addition to the other current measures.

Every collision that can be avoided at present could buy time for an eventual permanent solution to be implemented with the aid of future technological advances. Various other schemes have also been proposed which are outlined in Phipps et al. (2012) $)^{3}$ and references therein.

Solutions that involve perturbing the orbit of an object using a laser are appealing in that they eliminate the need to engineer technology that can chase debris, or even launch more spacecraft, as the laser source can be ground based. One way a laser could divert a piece of space debris is to ablate its surface with a high-energy light pulse to shift the object in the opposite direction to the removed particles. Laser ablation has been discussed for both ground based ${ }^{3-5}$ and space based sources. ${ }^{6,7}$ Another method which is being developed at Mount Stromlo Observatory is to use a continuous wave (CW) high-power laser (HPL), which has also been discussed in other articles. ${ }^{8-12}$ This method relies on photon pressure to transfer momentum to the object and its utilisation on Mount Stromlo will be discussed in this paper.

Section 2 details the theory behind the experiment to ensure that the space debris is exposed to enough photons. This is followed by an explanation of the adaptive optics (AO) system in Sec. 3 including how it operates, plus some performance tests on a steering mount. Sections 4 and 5 cover the progress of the laser guide star (LGS) and the high power laser (HPL) respectively prior to the conclusion.

\section{EXPERIMENTAL CONCEPT}

In order to have a chance at transferring non-negligible momentum to a piece of space debris, the object must be exposed to enough photon flux. For ground-based laser sources, several effects serve to disperse the beam as it propagates away from Earth's surface. Diffraction is inherent in wave field propagation and thus cannot be avoided. Usually discussed in the context of incoming light for imaging purposes, the smallest resolution a telescope can achieve is set by $\lambda / D$, where $\lambda$ is the wavelength of light and $D$ is the diameter of the telescope aperture. Therefore despite having a good beam quality provided by a fibre laser and a large sized telescope aperture, once a gaussian shaped beam reaches distances on the order of LEO altitudes, its spatial profile will have broader wings and a reduced maximum intensity.

The other effects to take into account are caused by the atmosphere. When propagating through the various gaseous layers, the laser power will be attenuated by absorption and scattering of photons from various atoms and molecules. On top of this the atmospheric turbulence layers which the laser must pass through to get to LEO will disperse the beam, known as atmospheric seeing. Turbulence can be caused by variations in temperature, pressure and humidity which leads to local density fluctuations in the air. This means that a beam of sufficiently large diameter will be propagating through an inhomogeneous medium across the wavefront as it encounters each layer of turbulence. With atmospheric seeing, the minimum resolution is limited by $\lambda / r_{0}$ where $r_{0}$ is the optical coherence length, also known as the Fried parameter.

While photon-atmosphere scattering cannot currently be prevented, fortunately the atmospheric seeing can be measured and counteracted using adaptive optics. ${ }^{13}$ Light coming from a distant object in space will have a plane wavefront until the point it enters Earth's atmosphere, upon which it then proceeds to experience the distortions. Adaptive optics takes advantage of a stable object like a star which is nearby in terms of angular distance and uses it as a reference source to measure the effect of turbulence. The final distorted wavefront from this source is measured using a wavefront sensor, which sends an error signal to a deformable mirror (DM). This serves to correct the optical path-length differences across the wavefront by varying the shape of the mirror so the reflected wavefront is flat again. Imaging the reference source during this process will sharpen the intensity distribution of the point spread function as it becomes almost diffraction limited. A depiction of an AO system taken from Rigaut (2015) ${ }^{14}$ is shown in Fig. 1.

Figure 2 shows a depiction of how Adaptive Optics Tracking and Pushing (AOTP) is to operate when viewed from outside of the telescope. The object is to be passively tracked using reflected sunlight during terminator., which is termed in this case as natural guide star (NGS) light. Due to the finite speed of the object, the HPL must point ahead of the object in order to make sure the pushing photons and the object cross paths. Higher order wavefront sensing using the NGS light would therefore result in an atmospheric correction for an angle 


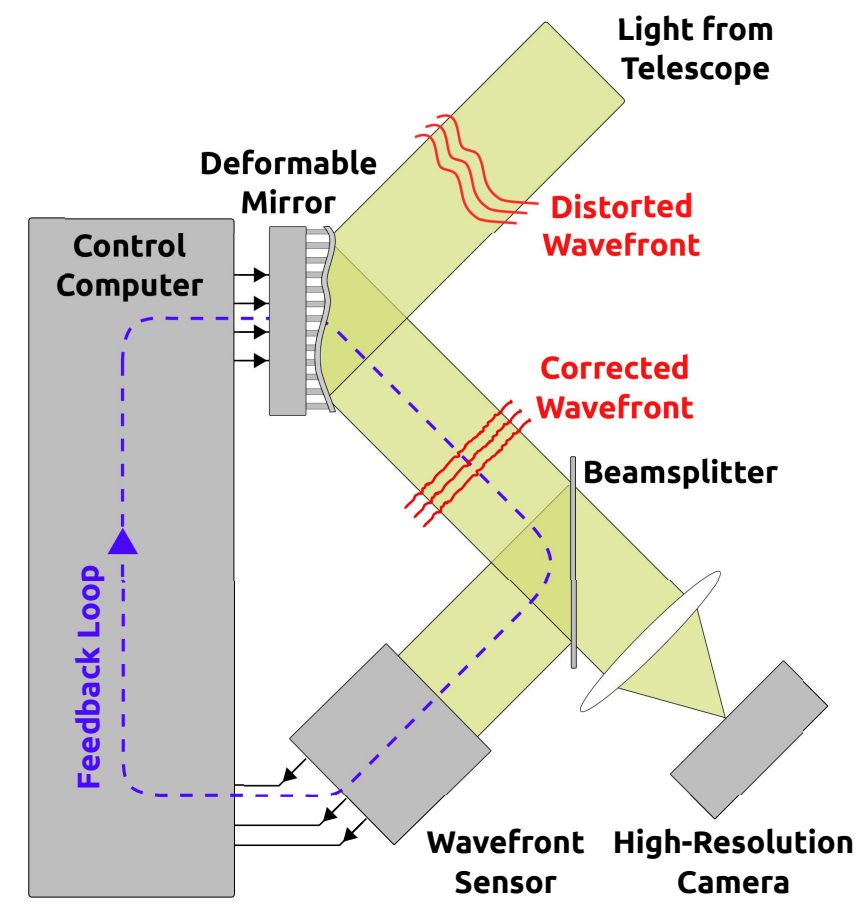

Figure 1. An example of an adaptive system taken from Rigaut $(2015)^{14}$

that the HPL has just passed through, so this issue will be overcome by using a laser guide star (LGS). Between $90-100 \mathrm{~km}$ in altitude a dilute layer of sodium exists which being an alkali atom, is easy to excite optically. A laser tuned to sodium's D2 transition can be propagated at an angle ahead of the object where absorption and then spontaneous emission of photons creates a source of scattered light. This "star" can be used as the reference source for wavefront sensing. As the scattered photons have a narrow range of wavelengths, when they are emitted back to the telescope they can then be filtered out easily and sent to a Shack-Hartmann wavefront sensor to close the AO feedback loop.

This experiment becomes possible through the aid of the LGS, as this artificial reference source can be positioned on-demand anywhere in the sky taking into account the physical constraints of the telescope, enabling it to follow a object. Importantly it can have a small point ahead angle with respect to the HPL to compensate for the time delay in the photon return and the AO loop. The inner workings of the AO system will be described in the next section.

\section{ADAPTIVE OPTICS SYSTEM}

This section delves into more detail about the adaptive optics system which is currently being developed at Mount Stromlo Observatory inside EOS's 1.8 meter telescope. The design concept has some similarities to the Adaptive Optic Demonstrator setup for laser ranging from a few years ago ${ }^{9}$ and indeed utilises the same experimental backbone. The optics setup is depicted in Fig 3.

From the Coudé path, incoming light from the telescope is reduced in diameter by two off-axis beam expanders, M1 and M2, before being directed onto the DM which is also mounted on a tip-tilt (TT) stage. The DM is a Xinetics model with 177 actuators making up a $15 \times 15$ array. Wavelengths below $950 \mathrm{~nm}$ then passes through a HPL dichroic and then the diameter is further reduced by lenses L1 and L2. Once reaching the LGS dichroic, a band of wavelengths between $589 \pm 20 \mathrm{~nm}$ are reflected at $45^{\circ}$ plus an angle between $0-0.4^{\circ}$ to take into account the point ahead angle of the laser guide star. The dichroic is mounted on a gimbal stage with stepper motors to automatically control this angle. Several more lenses reduce the beam diameter further before reaching the LGS wavefront sensor (LGSWFS). 


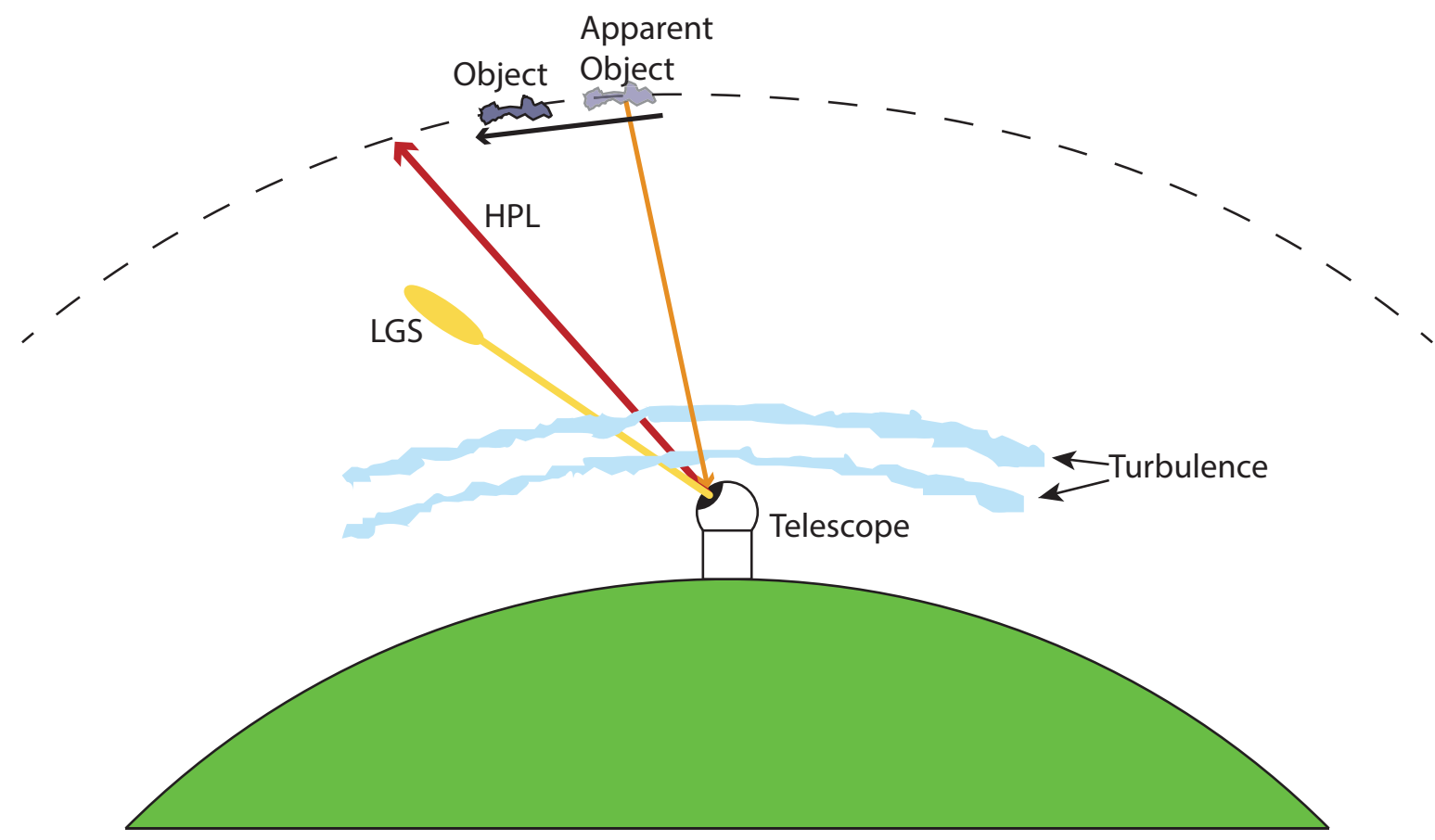

Figure 2. Not to scale drawing of the experiment from the perspective of outside the telescope.

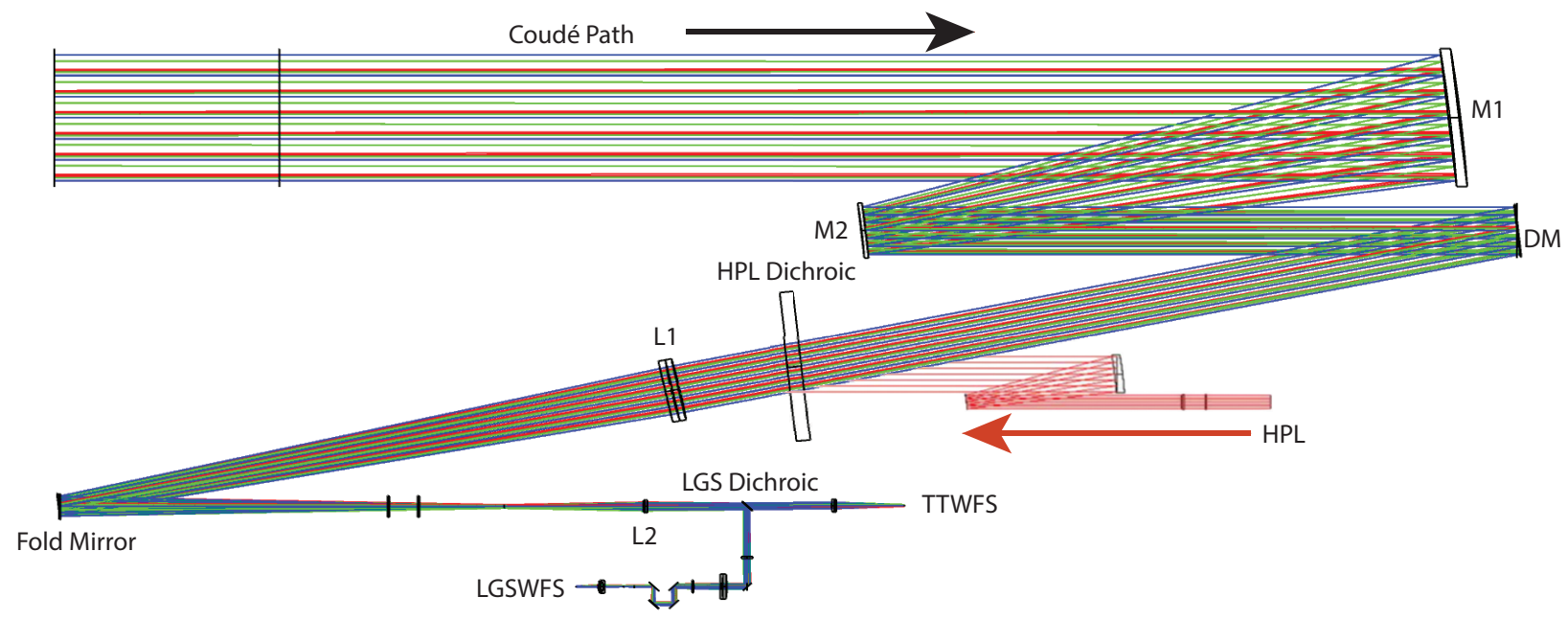

Figure 3. Zemax model of the AO system.

The first component of the LGSWFS the light passes through is a lenslet array which has $14 \times 14$ subapertures and a pitch of $300 \mu \mathrm{m}$. As the focal lengths of the lenslets are only $8.9 \mathrm{~mm}$ there is not enough space to image the focal spots directly onto the camera, therefore two relay lenses are used to project these focal spots onto the detector camera. The camera is an OCAM2k electron-multiplying CCD which runs at $2 \mathrm{kHz}$ to maintain the AO loop with the higher relative wind speeds associated with tracking a fast LEO object. The size and field of view of each subaperture on the camera will be $12+1$ pixels and $16.32 \operatorname{arcsec}$ respectively. The rest of the wavelengths pass through the LGS dichroic and are imaged on the tip-tilt wavefront sensor (TTWFS) which is an Andor 860 electron-multiplying CCD and operates at $500 \mathrm{~Hz}$.

HPL light enters the beam path by reflecting off the HPL dichroic and then onto the DM. Here the HPL beam is pre-conditioned by the DM in the AO system so once it has exited the telescope and propagated through 
the turbulence, the beam shape should have reverted back to an approximate gaussian shape with a small waist size.

\subsection{Operational Process}

The logistics behind this experiment are complex and require a high degree of automation in order to succeed.

The experimental sequence begins by selecting a suitable object. Ideally the object would have a high areato-mass ratio (HAMR) and have a path located within the capabilities of the experiment. The lowest elevation angle with enough photon return from the LGS is $30^{\circ}$ while the maximum elevation angle is limited by the telescope dome vignetting the guide star laser (GSL) at $76^{\circ}$. The minimum altitude of the object is $500 \mathrm{~km}$, limited by the performance of the AO system while the maximum height of the object is $1500 \mathrm{~km}$ due to the resolving power of the $\mathrm{AO}$ system to provide enough photon pressure. With respect to the telescope, exposing the object with photons during its rise to the zenith will have the effect of reducing its kinetic energy. Conversely doing the same past the zenith will have the opposite effect. Therefore exposure of the object during its entire path across the sky is counterproductive, so in this experiment the laser will only run during the object's journey beyond the zenith. From an orbital dynamics perspective because this is the direction that the photons are traversing, the perturbation will be enhanced slightly compared to the contrary scenario.

In an experiment where every second that can be dedicated to pushing the object is vital, instrumentation and automated processes will need to enable a fast transition from object location through to the pushing stage. The object will firstly be acquired on a wide-field camera (WFC) which has a field of view of $1^{\circ}$ and will correct the pointing of the telescope within $12 \mathrm{~s}$ of acquisition. Once the telescope is tracking, the TTWFS receives a signal and sends it to the real-time computer (RTC) which then closes a loop with the telescope pointing and the TT stage.

A shutter will then open to allow the guide star laser (GSL) to propagate and the subsequent LGS should be within the field of view of the LGSWFS. A TT measurement can then be calculated on the LGSWFS and this signal can be used to modulate a beam pointing mirror in the LGS facility to correct the position of the LGS. The mount holding the LGS dichroic has steering capabilities so it will govern the point ahead angle of the LGS through a pre-calculation depending on the trajectory of the object. At this point, the higher order wavefront coming from the LGS can be measured on the LGSWFS and this signal is repackaged by the RTC to adjust the DM and compensate for the atmospheric distortions. Once all of the loops are closed, the HPL can be introduced to start the engagement process.

With so many components it is important that every one of them is tested prior to being added into the apparatus. One recent example of this is presented in the next subsection.

\subsection{Gimbal Mount Testing}

This section provides some details on the testing of the gimballed mount that holds the steering LGS dichroic mirror and its role is described in the previous section. The rear plate of the mount is fitted with stepper motors which are controlled by the RTC and serve to drive the front plate accordingly. The angle of the light reflecting off the dichroic during tracking will be calculated prior to tracking as the approximate trajectory of the object is known beforehand.

The mount in question is the U200-G from Newport and the stepper motors are NSA12, also from Newport. Like many standard kinematic mirror mounts, it has two degrees of freedom: the horizontal axis $(x)$ and the vertical axis $(y)$. As wavefront measurements are sensitive, driving the motors should enable jitter-free movement and the two axes should be isolated from one another.

The result shown in Fig. 4 is the image of a fibre source on a camera after being reflected off the dichroic. The mirror is driven in a circular motion for a total time of $350 \mathrm{~s}$, a similar time span to the time of engagement.

There are several noteworthy factors here including the drift in central position of the ellipse upon each cycle. This is likely caused by the steady heating of the stepper motors over the duration in which they operate. This could be mitigated by initiating warm up cycles prior to starting the experiment which would allow the motors to reach equilibrium. 


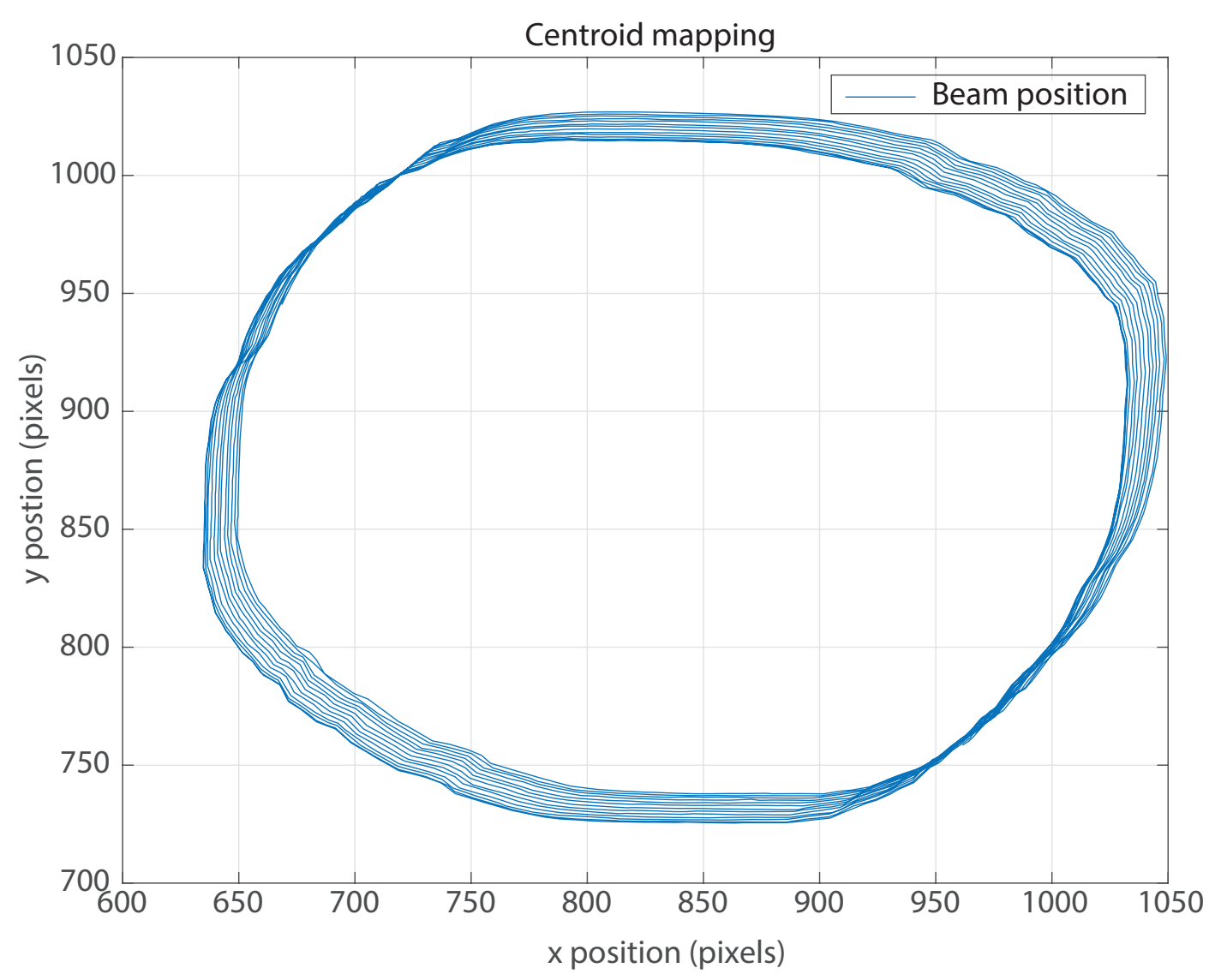

Figure 4. Centroid map showing beam position when steered in circular motion.

The other issue is prevalent while both axes are being considerably driven at the same time. Certain "bumps" can be observed and are repetitive upon each cycle. Other results also show that driving one axis causes a nonnegligible shift in the other and this is due to the front and rear plates misaligning with respect to each other which therefore shifts the centre of rotation of the mount. Due to these tests, investigations are now underway with a new mount.

\section{LASER GUIDE STAR FACILITY}

The top-level requirements for the Laser Guide Star Facility (LGSF) that will equip the EOS $1.8 \mathrm{~m}$ telescope at Mount Stromlo Observatory are partially derived from ${ }^{15}$ and are summarized in Tab. 1.

The LGSF system architecture is presented in schematic form in Fig 5. The Laser Guide Star Facility includes four major subsystems: • The Guidestar Laser (GSL) • The Beam Transfer Optics (BTO) • The Laser Launch Telescope (LLT), and $\bullet$ The Laser Safety Systems as well as associated electronics and software control systems to operate them. A top-level LGSF Control System encapsulates those control systems to interface with the AOTP and telescope control systems.

EOS Space Systems (EOS) is responsible for the design, fabrication and installation of the Guidestar Laser and Laser Safety Systems, and the Australian National University (ANU) is responsible for the design, fabrication and installation of the BTO and LLT, as well as the LGSF Control System. All LGSF subsystems are currently in the fabrication phase and will be installed on the EOS $1.8 \mathrm{~m}$ telescope over the 2018 winter period in Canberra. Integration and testing of each component on the telescope is foreseen to take place in Q3 2018. This will be 
Table 1. LGSF top-level requirements.

\begin{tabular}{|l|l|}
\hline Performance Parameter & Requirement \\
\hline \hline Type and number of LGS & 1 sodium LGS \\
\hline LGS point ahead angle & $0-10 \operatorname{arcsec}$ \\
\hline LGS photon return on the ground & 1500 photons $/ \mathrm{cm}^{2} / \mathrm{s}$ \\
\hline LGS spot size on LGS WFS subaperture at zenith & $1.6 \operatorname{arcsec}$ \\
\hline LGS projection system focusing accuracy & $0.1 \operatorname{arcsec}$ \\
\hline LGS steering range & $135 \operatorname{arcsec}$ \\
\hline LGS blind pointing accuracy & $2 \operatorname{arcsec}$ \\
\hline LGS residual on-sky jitter at $200 \mathrm{~Hz}$ & $0.1 \operatorname{arcsec} \mathrm{rms}$ \\
\hline
\end{tabular}

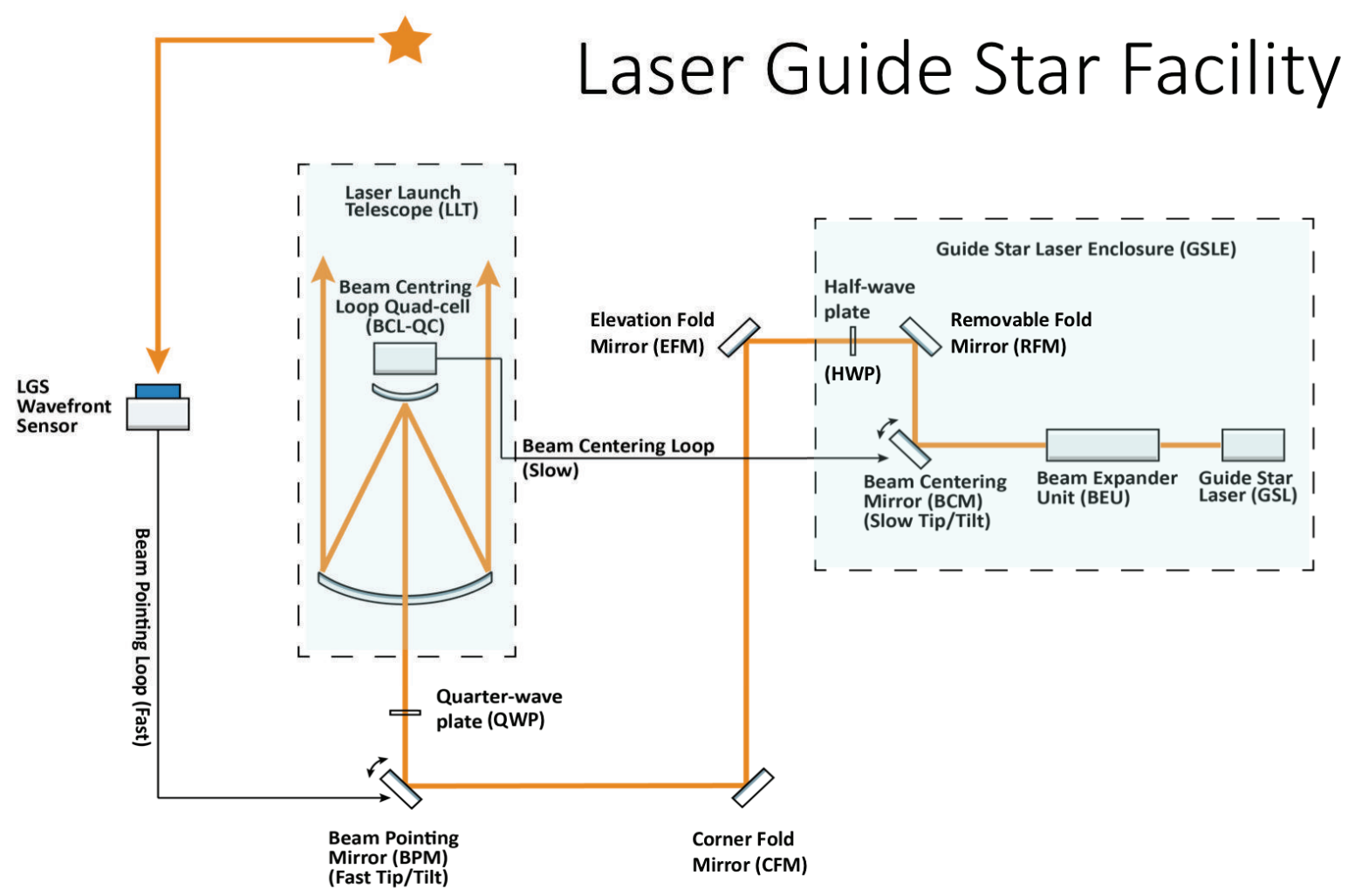

Figure 5. Laser Guide Star Facility for the Mount Stromlo EOS Laser Tracking Station 1.8m telescope.

followed by system-level integration of the Laser Guide Star Facility in Q4 2018 and on-sky commissioning in Q1 2019. When the Guidestar Laser is propagated on sky over Mount Stromlo, it will be the first time that a Laser Guide Star is created in Australian skies.

\subsection{Guidestar Laser}

The guide star laser enclosure incorporates two $589 \mathrm{~nm}$ laser sources, one to be integrated late 2018 and the other early 2019. The former system is a sum-frequency solid state architecture to be delivered by EOS Space Systems and discussed in this section. The latter source is a novel semiconductor design, currently being built 


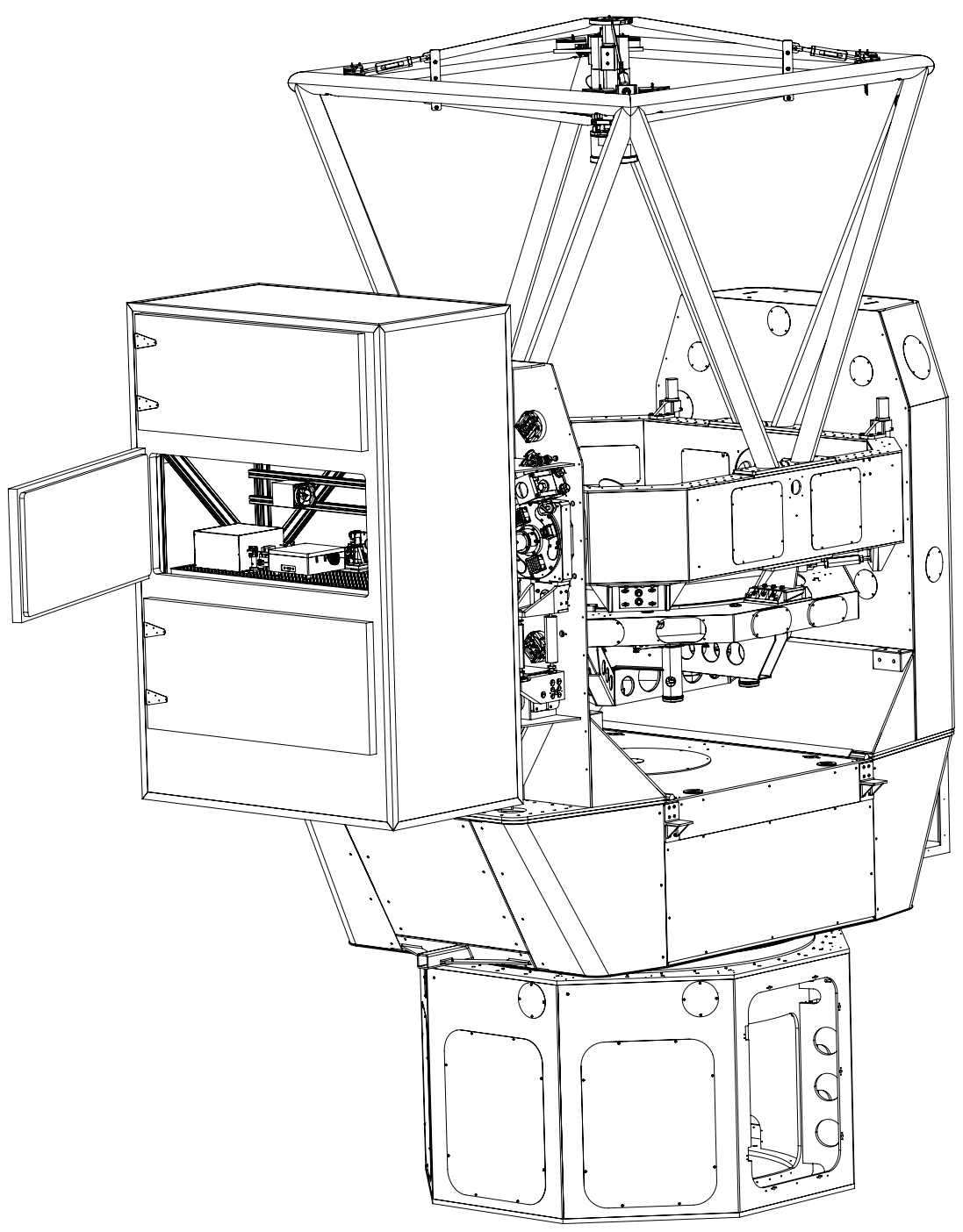

Figure 6. Guidestar Laser Enclosure, BTO and LLT (behind Guidestar Laser Enclosure) mounted on EOS 1.8m telescope.

by US laser manufacturer Areté Associates in partnership with ANU, EOS Space Systems, Lockheed Martin Space Systems and other Australian and US academic and industry partners. ${ }^{16}$ The GSL system is designed to allow the laser sources to be propagated either independently or simultaneously. The EOS GSL consists of two $30 \mathrm{~W}$ infrared lasers, non-linearly combined in a sum-frequency generator (SFG) stage to produce the desired $589 \mathrm{~nm}$ output with a power also approaching $30 \mathrm{~W}$. The two infra-red lasers operate at $1342 \mathrm{~nm}$ and $1050 \mathrm{~nm}$ wavelength, both in a master-oscillator-power-amplifier (MOPA) configuration. The grating-tuned $1342 \mathrm{~nm}$ oscillator is amplified by a commercial Raman amplifier and a series of neodymium yttrium vanadate stages. The $1050 \mathrm{~nm}$ laser is entirely fibre-based using ytterbium doped fibres as the gain media. The SFG is a doubly resonant cavity enclosing a barium borate crystal to perform the non-linear wavelength conversion. A sample of the SFG output is directed to a sodium vapour cell for wavelength locking with the remainder transmitted through an electro-optic-modulator (EOM) to permit the addition of $1.7 \mathrm{GHz}$ modulation sidebands to the laser output spectra. These sidebands drive the "repumping" mechanism ${ }^{17}$ used to enhance the return photon flux from the mesospheric sodium layer. The laser system is internally controlled by a distributed real-time Linux controller, which permits integration with the observatory control systems (OCS) and the laser safety systems described in Section 6. 


\subsection{Beam Transfer Optics and Laser Launch Telescope}

Part of the Beam Transfer Optics (BTO) will be mounted within the Guidestar Laser Enclosure (GSLE), on the laser optical bench hosting the $589 \mathrm{~nm}$ sum frequency generator (Figs. 6 and 7).

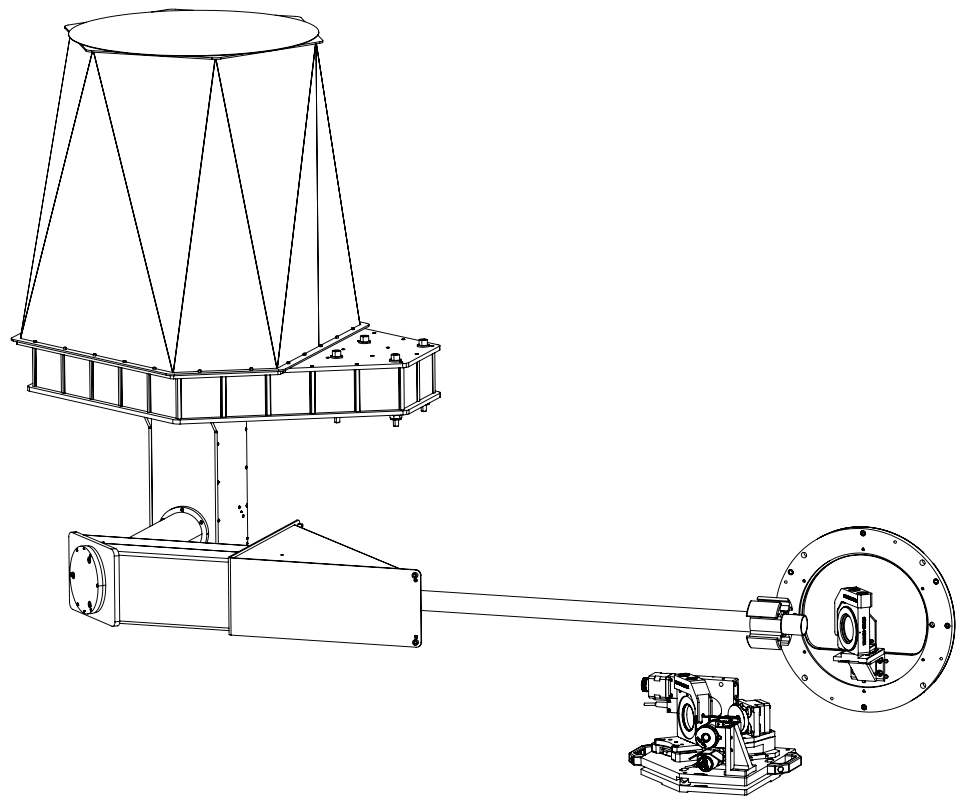

Figure 7. Closer view but same angle Fig. 6 except showing only BTO and LLT.

The GSLE sits in a gravity invariant environment at the $1.8 \mathrm{~m}$ telescope Nasmyth port, and rotates in azimuth with the telescope. A Beam Expander Unit (BEU) will first expand the laser output beam from its nominal, gaussian $2.8 \mathrm{~mm}$ diameter at $1 / e^{2}$ intensity points to a $10 \mathrm{~mm}$ diameter at $1 / e^{2}$ intensity points. A couple of fold mirrors (including the Beam Centering Mirror (BCM), and a Removable Fold Mirror (RFM) providing access to the telescope elevation axis for non-laser alignment work) will then direct the beam out of the GSLE, through a window, along the telescope elevation axis, and into the $1.8 \mathrm{~m}$ primary mirror cell.

The Elevation Fold Mirror (EFM) is the first BTO component in the remainder of the BTO and LLT optical train to be mounted on the EOS telescope and thus to be moving in elevation with it. EFM directs the laser beam through the EOS telescope primary cell towards the Corner Fold Mirror (CFM) located on the exterior of the primary cell wall. CFM redirects the beam towards the Beam Pointing Mirror (BPM) which is mounted underneath the LLT. BPM sends the beam upward into the 20X LLT which expands the beam diameter to $200 \mathrm{~mm}$ at $1 / e^{2}$ intensity points and projects the guidestar laser beam on sky. Together, BCM and BPM provide centering and pointing of the laser beam on the LLT secondary mirror and on the sky respectively. Centering of the laser beam on the LLT secondary mirror (the optical stop of BTO+LLT system) is measured via laser light leaking through the LLT secondary mirror onto a Position Sensing Device (PSD) at a slow rate of $\sim 10 \mathrm{~Hz}$. LGS pointing errors on sky are measured by the LGSWFS at a higher rate of $\sim 200 \mathrm{~Hz}$. Centering and pointing errors are processed by the LGSF control system to control BCM and BPM in a coordinated fashion such that BCM primarily compensates for slow centering errors in the BTO+LLT beam train while BPM primarily compensates for pointing errors on-sky. BPM also provides on-sky laser beam steering and maintains the required point-ahead angle as directed by the LGSF Control System. Finally, polarization control is provided via a pair of wave plates whose purpose is to deliver circular polarization on-sky so as to optimise the LGS photon return for all telescope elevation angles. The linear polarization out of the Guidestar Laser is maintained throughout the BTO thanks to a Half-Wave Plate (HWP) that is placed near the output of the GSLE. This HWP is mounted within a rotation stage that follows the telescope elevation angle via a Look-Up Table (LUT), thus ensuring that the laser polarization remains constant on all subsequent optics in the BTO and LLT optical train. When used in 
combination with the fixed Quarter-Wave Plate (QWP) mounted at the input of the Laser Launch Telescope (LLT), the HWP rotation ensures that the projected laser beam polarization remains circular on sky at all times.

\section{HIGH POWER LASER}

Since 2003 a high average power laser program has been running with the primary objectives of developing laser systems capable of generating multi-kilo-watt average power output with other key specifications that can meet the performance requirements for space related applications, including ranging, tracking, and space debris manoeuvre. The key specifications include mode structure, beam pointing stability, and most importantly beam quality. The pulsed system should be able to generate multi-Joule pulse energy with nano-second pulse width and at a pulse repetition rate between 100-200 Hz. The CW system should be able to generate a single mode beam with output power close to $10 \mathrm{~kW}$. The pulsed system developed has achieved $4.7 \mathrm{~J}$ pulse energy at $170 \mathrm{~Hz}$ while maintaining a good beam quality $M^{2} \sim 3$. All the key specifications achieved are shown in Tab. 2 .

Table 2. Key specifications achieved.

\begin{tabular}{|l|l|}
\hline Parameters & Specification Achieved \\
\hline \hline Pulse Energy $(\mathrm{J})$ & 4.7 \\
\hline Repetition Rate $(\mathrm{Hz})$ & 170 \\
\hline Average Power $(\mathrm{W})$ & 800 \\
\hline Pulse Width $(\mathrm{ns})$ & 20 \\
\hline Beam Quality & $M_{x}^{2}: 2.64, M_{y}^{2}: 3.06$ \\
\hline Beam Pointing Stability & $0.7 \operatorname{arcsec}$ \\
\hline
\end{tabular}

So far two versions, a tabletop version and a modular version have been designed, developed, and in service for more than two years with excellent performance and reliability. ${ }^{18}$ The tabletop version is suitable for the laboratory environment. The modular version, as shown in Fig 8, with a 'front-end', pre-amplifier, power amplifier, and beam shaping and diagnostic modules offers flexibility, easy transportation and field deployment.

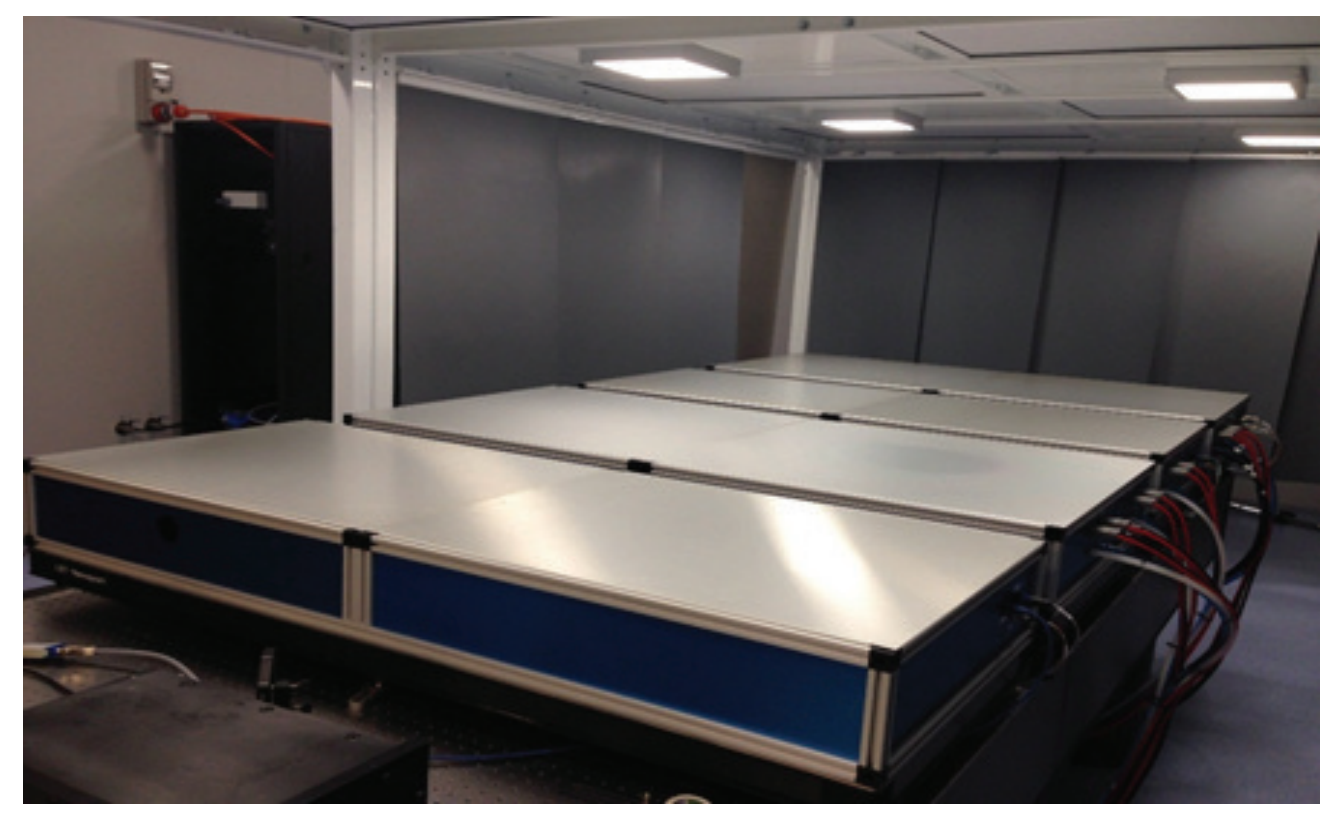

Figure 8. Modular Version. 
The systems have the capacity to support fully automated 24/7 unmanned operations and have been used for ranging and tracking space debris successfully.

Investigations on using pulsed and CW lasers for space debris manoeuvre through laser ablation and photon pressure processes have been conducted at EOS. Laser ablation occurs under the radiation of high energy laser beam where a part of the space debris material can be ablated and provides an impulse to the space debris. Photon pressure is a result of the photon momentum transfer. If a piece of debris absorbs or reflects incoming photons, the momentum transferred leads to a small but noticeable force.

For CW systems, some different approaches including thin disk and fibre have been investigated. For the thin disk approach, more than $4000 \mathrm{~W}$ multi-mode power output has been obtained from a single $40 \mathrm{~mm}$ diameter Yb:YAG thin disk. The fibre approach has different schemes including Coherent Beam Combination (CBC) and Wavelength Beam Combination (WBC), both of which have been evaluated. As a result, a baseline system which is technologically practical and financially realistic has been proposed and a 2-phase approach has been planned. In Phase-1 $8 \mathrm{~kW}$ output power is going to be obtained through WBC scheme. In Phase-2 more than $15 \mathrm{~kW}$ through combining $8 \mathrm{~kW}$ laser beam with the output beams from other multi-kilo-Watt laser sources, such as another fibre laser or thin disk laser is going to be obtained.

Significant progress has been made on the development of key technologies and assemblies such as fibre based single frequency seed oscillator. The seed oscillator is based on a stable broadband Amplified Spontaneous Emission (ASE) source with no feedback cavity. It emits a broadband continuous spectrum, then spectral filters are used to select the laser wavelength and control the spectral linewidth as required. After these, a fibre amplifier is used to amplify the laser output to the required power level. The seed oscillator can generate $10 \mathrm{~mW}$ output power with a spectral linewidth in the range of 0.1-0.8 nm according to the requirement of $2 \mathrm{~kW}$ fibre amplifier modules. So far four seed oscillators with four output wavelengths shown in Tab. 3 have been developed and thoroughly tested.

Table 3. Output wavelengths of four seed oscillators.

\begin{tabular}{|l|l|}
\hline Oscillator-1 & $1058 \mathrm{~nm}$ \\
\hline Oscillator-2 & $1060 \mathrm{~nm}$ \\
\hline Oscillator-3 & $1062 \mathrm{~nm}$ \\
\hline Oscillator-4 & $1064 \mathrm{~nm}$ \\
\hline
\end{tabular}

Both output power and spectrum are very stable and can meet the requirement for seeding the $2 \mathrm{~kW}$ fibre amplifiers. $1958 \mathrm{~W}$ output power has been obtained from amplifier channel-1 at $1064 \mathrm{~nm}$. Further development and testing of the remaining three amplifier channels are in progress.

\section{SAFETY CONTROL}

This section covers the basic control measures at the Mount Stromlo Space Research Centre. These fall into three categories which include engineering control, personal protective equipment, and administrative and procedural controls.

\subsection{Engineering Control}

Many of the engineering controls are referred to as performance requirements. These standards are reviewed below:

1. Protective Housing. An enclosure about the laser which prevents access to radiant power at levels higher than the intended system classification. A protective housing is required for all Class 3 and 4 lasers, and an interlock must be provided on the housing of all Class 3 and 4 lasers that actuates on removal or displacement of the protective housing to prevent exposure to greater than Maximum Permitted Exposure (MPE) levels. In some cases a properly interlocked room can be considered the protective housing for an open laser. 
2. Key Switch Interlocks. All Class 4 lasers and systems shall have a removable key and switch master interlock, and the laser shall not be capable of operation when the key is removed. Only authorised system operators shall have possession of the key. Interlocks should also be incorporated with beam shutters when optical viewing systems such as telescopes or microscopes are used to view the beam or reflected beam under potentially hazardous conditions. Optical interlocks are required for all Class 3 and 4 laser systems.

3. Beam Path Enclosures. Some users of Class 3 and 4 laser and systems dictate that the beam path be enclosed. It is recommended that such enclosures be equipped with appropriate safety interlocks to prevent operation unless the enclosures are properly installed and all means of operator access secured.

4. Beam Stops or Attenuators. Class 3 and 4 lasers and systems should be provided with a permanently attached beam stop or attenuator that reduces the output emission to a level at or below the appropriate MPE level when the laser system is at Standby.

5. Visible or Audible Warning Systems. An audible tone and/or visual warning such as a flashing light is recommended as an area control for Class 3 and 4 laser operations. Such warning devices are to be activated upon system start up and are to be uniquely identified with the laser operation.

6. Viewing Optics and Windows. All viewing portals, optics, windows or display screens included as an integral part of an enclosed laser or system shall incorporate some means to attenuate the radiation through the portal to levels below the appropriate MPE levels, or incorporate interlocks that will suspend hazardous laser emissions.

7. Interlock Requirements. Adjustment or procedures during service shall not cause any safety interlocks to become inoperative, or the laser radiation outside the protective housing to exceed the MPE limits, unless a temporary laser controlled area is established. Interlocks of pulsed lasers or systems shall be designed so as to prevent laser operation when the interlock is closed by such methods as dumping stored capacitor bank energy into a dummy load. Interlocks for Class 3 and 4 lasers or systems should be provided with a remote interlock connector to allow electrical connection to an emergency disconnect interlock or to room, door, or fixture interlocks.

8. Aircraft Detection Camera (ADC). An independent 3-5 $\mu \mathrm{m}$ midwave infrared camera with a field of view parallel to and overlapping the primary (laser) optical axis is permanently mounted on the telescope. This camera inhibits all laser emissions if any object higher in temperature than the sky background enters the frame. An example image is shown in Fig. 9 of an aircraft at cruising altitude passing through the field of view.

9. Aircraft Transponder. Aviation law requires increasing numbers of aircraft to carry ADS-B (Automatic Dependant Surveillance Broadcast) transponders that periodically broadcast the planes' position via an unencrypted radio transmission. The observatory has a receiver that constantly monitors the broadcast positions of surrounding aircraft and inhibits laser emissions if the current position of the projected track of an aircraft comes within determined limits of the beam location.

\subsection{Laser controlled area}

When the entire beam path from a Class 3 or 4 laser is not sufficiently enclosed or baffled such that access to radiation above the MPE level is possible a laser controlled area shall be established. The requirements for such an area are that it shall:

1. Have direct supervision of the Laser Safety Officer or his delegate/shift leader during all times of operation.

2. Require approved entry of any non involved personnel.

3. Be posted with the appropriate warning signs.

4. Terminate all potentially hazardous beams. 


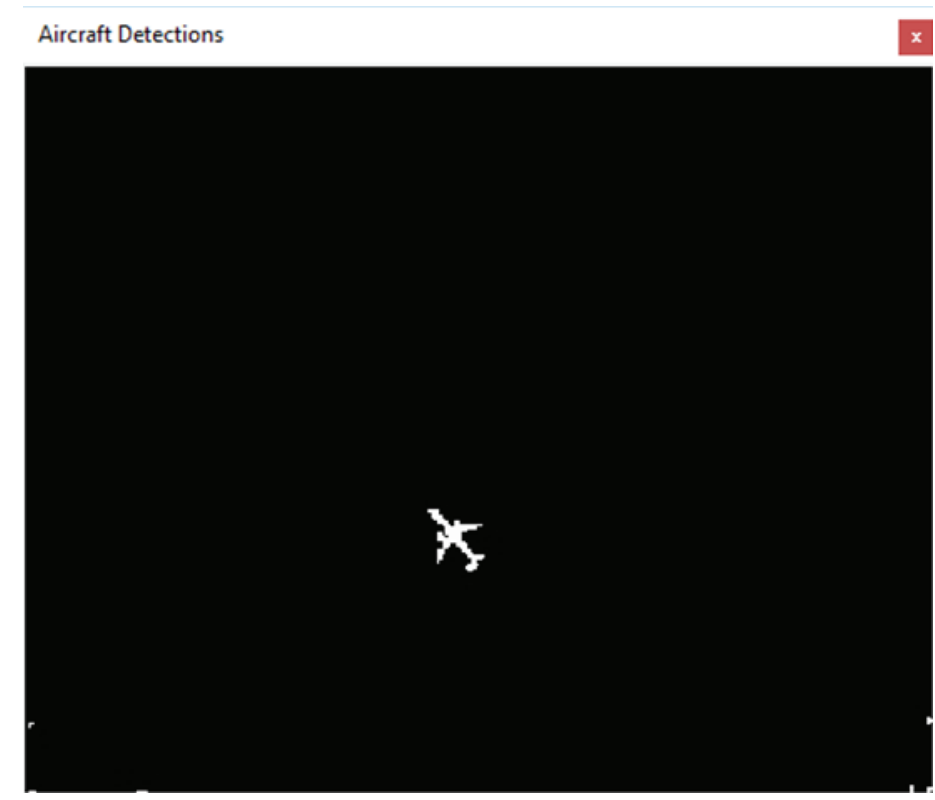

Figure 9. Example image of aircraft detection.

5. Use diffusely reflecting materials near the beam.

6. Incorporate appropriate safety interlocks at doorways. Such latches shall at all times allow for egress by those in the laser-controlled area.

7. Authorised personnel may enter the area if there is no hazard at the entry point and if the appropriate eye protection is worn.

8. Persons with only an operational familiarity of the laser system shall not under any circumstances attempt any maintenance on the laser system or associated optics.

9. Maintenance on the laser system shall only be carried out by fully trained persons within the hazard area.

\subsection{1.2 Laser warning signs and labels}

Lasers and systems shall be properly labelled in accordance with the appropriate safety standards AS/NZS 2211.1. Area posting is required for Class 3 and 4 lasers and systems. All signs and labels associated with Class 3 and 4 lasers and systems are to show the word DANGER.

\subsection{Personal protection equipment}

Personal protective equipment for laser safety generally means eye protection in the form of goggles or special prescription eyewear using special high optical density filter materials to reduce the potential ocular exposure to below the MPE limit. A wide variety of commercially available optical filter glass and plastic is available for laser eye protection - some available in eye spectacles ground to prescription specifications. One filter type may be applicable to more than one wavelength. As well as eye protection personal protection is also provided in the form of overalls, disposable rubber gloves and respiratory face masks. Proper chemical glassware and accessories are provided for handling toxic chemicals associated with the laser system.

\subsection{Administrative and procedure controls}

The overall laser safety programme is administered by the Laser Safety Officer. His/her principle responsibility is to establish and periodically review control measures, and to provide training in all aspects of laser safety. 


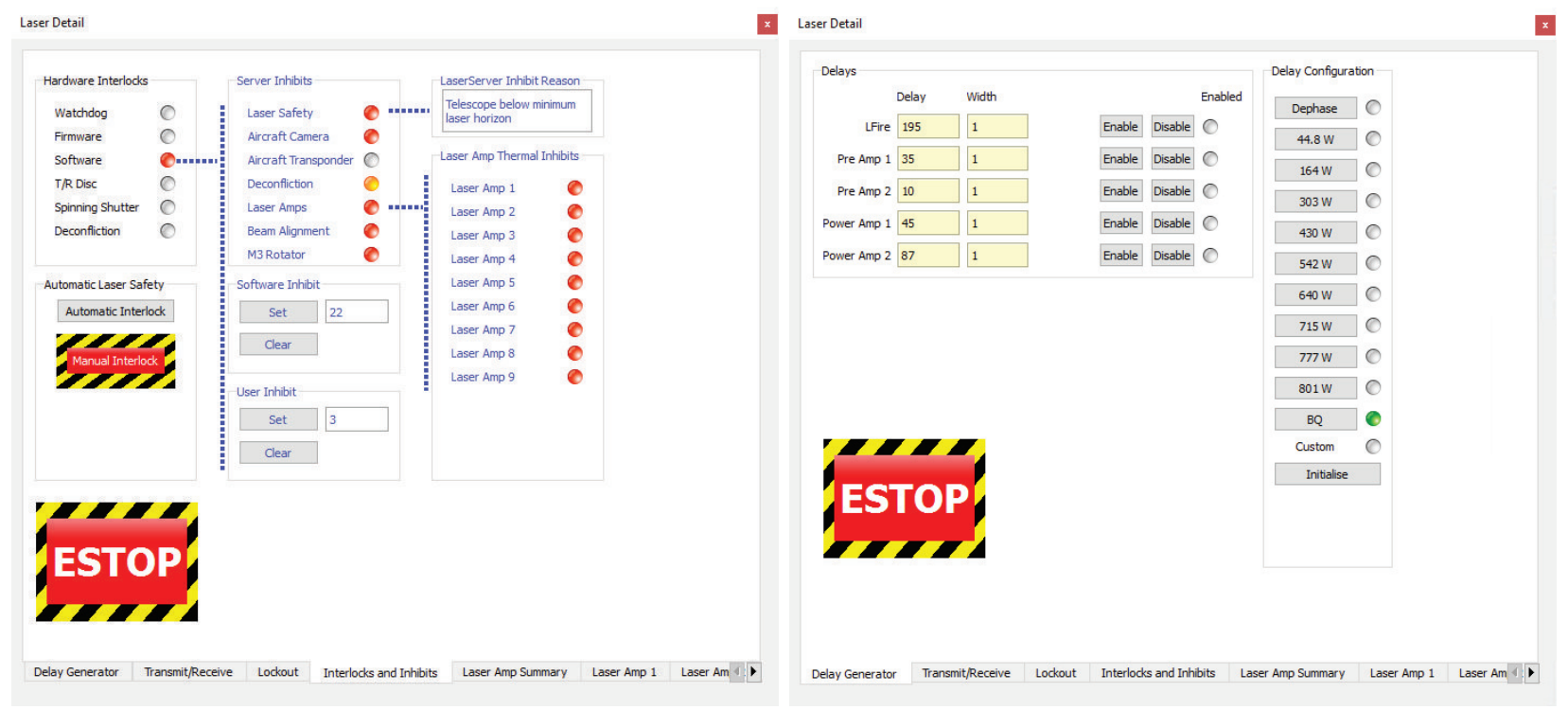

Figure 10. Typical laser GUI

\section{IMPLEMENTATION}

As there are many moving parts to this experiment, it is important to test their functionality in a logical way and thus implementation has been divided into several phases. Therefore once various hardware and software constituents have been set up, they will be tested methodically to ensure smooth execution. Factors such as time, safety and availability of equipment were also taken into account when planning each phase and these are briefed in Tab. 4.

Table 4. A table outlining the various phases of implementation.

\begin{tabular}{|l|l|l|}
\hline Phase & Timeline & Stage of implementation \\
\hline \hline 1 & Q3 2018 & Test telescope tracking and AO on sidereal object \\
\hline 2 & Q4 2018 & Passively track LEO object \\
\hline 3 & Q2 2019 & Test AO system with LGS on a sidereal and then a LEO object \\
\hline 4 & Q2 2019 & Introduce a low power laser, test AO performance on high altitude platform \\
\hline 5 & Q2 2019 & Introduce HPL, test AO performance, engage object \\
\hline
\end{tabular}

As it stands the majority of hardware and software has been implemented and developed. Commencement of Phase 1 is to begin in Q3 2018 and the aim is to engage and object during Q2 2019.

\section{CONCLUSION}

In an effort to mitigate collisions in low earth orbit, an adaptive optics system is being built at Mount Stromlo Observatory. This will take advantage of a laser guide star as a reference source to correct for atmospheric distortions and a high power laser to transfer photon momentum to the space debris. As there are many moving parts to this experiment, it is important to test their functionality in a logical way and thus implementation and automation will be divided into various phases. Therefore once various hardware and software constituents have been set up, they will be tested methodically to ensure smooth execution. The performance of the AO system is to be evaluated through a photon flux measurement on a payload during Q2 2019. 


\section{ACKNOWLEDGEMENTS}

The authors would like to acknowledge the support of the Cooperative Research Centre for Space Environment Management (SERC Limited) through the Australian Government's Cooperative Research Centre Programme.

\section{REFERENCES}

[1] Liou, J.-C., "Engineering and technology challenges for active debris removal," Progress in Propulsion Physics 4, 735-748 (2011).

[2] Kessler, D. J. and Cour-Palais, B. G., "Collision frequency of artificial satellites: The creation of a debris belt," Journal of Geophysical Research: Space Physics 83(A6), 2637-2646 (1978).

[3] Phipps, C. R., Baker, K. L., Libby, S. B., Liedahl, D. A., Olivier, S. S., Pleasance, L. D., Rubenchik, A., Trebes, J. E., Victor George, E., Marcovici, B., Reilly, J. P., and Valley, M. T., "Removing orbital debris with lasers," Advances in Space Research 49(9), 1283-1300 (2012).

[4] Bohn, W. L., "Pulsed coil for space debris removal," in [Optoelectronics '99 - Integrated Optoelectronic Devices], 3612, 6, SPIE (1999).

[5] Gehly, S., Bennett, J., and Afful, M., "Lp-norm batch estimation as applied to orbit determination," in $[A I A A / A A S$ Astrodynamics Specialist Conference], AIAA SPACE Forum, 5568, American Institute of Aeronautics and Astronautics (2016).

[6] Choi, S. H. and Pappa, R. S., "Assessment study of small space debris removal by laser satellites," Recent Patents on Space Technology 2(2), 116-122 (2012).

[7] Shen, S., Jin, X., and Hao, C., "Cleaning space debris with a space-based laser system," Chinese Journal of Aeronautics 27(4), 805-811 (2014).

[8] Mason, J., Stupl, J., Marshall, W., and Levit, C., "Orbital debris-debris collision avoidance," Advances in Space Research 48(10), 1643-1655 (2011).

[9] Bennet, F., d'Orgeville, C., Gao, Y., Gardhouse, W., Paulin, N., Price, I., Rigaut, F., Ritchie, I. T., Smith, C. H., Uhlendorf, K., and Wang, Y., "Adaptive optics for space debris tracking," in [SPIE Astronomical Telescopes + Instrumentation], 9148, 9, SPIE (2014).

[10] Yang Yang, F., Nelson, B., Aziz, J., Carlino, R., Dono Perez, A., Faber, N., Foster, C., Frost, C., Henze, C., Karacalığlu, A. G., Levit, C., Marshall, W., Mason, J., O’Toole, C., Swenson, J., Worden, S. P., and Stupl, J., "Lightforce photon-pressure collision avoidance: Efficiency analysis in the current debris environment and long-term simulation perspective," Acta Astronautica 126, 411-423 (2016).

[11] Zovaro, A., Bennet, F., Copeland, M., Rigaut, F., d'Orgeville, C., Grosse, D., and Bold, M., "Harnessing adaptive optics for space debris collision mitigation," in [Advanced Maui Optical and Space Surveillance Technologies Conference], (2016).

[12] Grosse, D., Bennet, F., Copeland, M., d'Orgeville, C., Rigaut, F., and Price, I., "Adaptive optics for satellite imaging and earth based space debris manoeuvres," in [Proceedings of the 7th European Conference on Space Debris], 7 (2017).

[13] Hardy, J. W., [Adaptive optics for astronomical telescopes], vol. 16, Oxford University Press on Demand (1998).

[14] Rigaut, F., "Astronomical adaptive optics," Publications of the Astronomical Society of the Pacific 127(958), 1197 (2015).

[15] d'Orgeville, C., Bennet, F., Blundell, M., Brister, R., Chan, A., Dawson, M., Gao, Y., Paulin, N., Price, I., Rigaut, F., Ritchie, I., Sellars, M., Smith, C., Uhlendorf, K., and Wang, Y., "A sodium laser guide star facility for the anu/eos space debris tracking adaptive optics demonstrator," in [SPIE Astronomical Telescopes + Instrumentation], 9148, 16, SPIE (2014).

[16] d'Orgeville, C. and Fetzer, G. J., "Four generations of sodium guide star lasers for adaptive optics in astronomy and space situational awareness," in [SPIE Astronomical Telescopes + Instrumentation], 9909, 18 (2016).

[17] Lihang, L., Wang, H., Hua, W., Ning, Y., and Xu, X., "Fluorescence enhancing mechanism of optical repumping in sodium atoms for brighter laser guide star," Optics Express 24(7), 6976-6984 (2016).

[18] Gao, Y., Wang, Y., Chan, A., Dawson, M., and Greene, B., "High average power diode pumped solid state laser," Laser Physics Letters 14(3), 035803 (2017). 